\title{
Effects of different anesthesia protocols on lactation in the postpartum period
}

\author{
Leyla Kutlucan ${ }^{1}$, İknur S. Seker ${ }^{1}$, Yavuz Demiraran', Özlem Ersoy ${ }^{1}$, İbrahim Karagöz', Gülbin Sezen ${ }^{1}$, Seyit Ali Köse ${ }^{2}$ \\ 'Department of Anesthesiology, Düzce University Faculty of Medicine, Düzce, Turkey \\ ${ }^{2}$ Department of Obstetrics and Gynecology, Süleyman Demirel University Faculty of Medicine, Isparta, Turkey
}

\section{Abstract}

Objective: Many factors can influence the secretion of breast milk. Cesarean section is a risk factor for late onset of breastfeeding.

Material and Methods: In our study, we compared the lactation process by mothers who underwent elective cesarean section under general anesthesia, spinal anesthesia, epidural anesthesia, and normal birth; 84 patients between 18-40 years of age with a risk of ASA I-II were included. Randomly patients were divided into four groups: group G (general anesthesia, n:21), group S (spinal anesthesia, n:21), group E (epidural anesthesia, n:21), and group V (vaginal birth, without anesthesia, n:21). Oxytocin and prolactin values of all patients before and after operation or birth were recorded. In addition the initiation time of lactation after delivery or cesarean section were recorded.

Results: In all groups, there were no significant differences among hormone levels in the prepartum period $(p=0.350)$. Prolactin levels in group $\mathrm{G}(\mathrm{p}=0.011)$ and oxytocin levels in group $\mathrm{V}(\mathrm{p}=0.012)$ in the postpartum period were significantly higher than in the other groups. The start of lactation was significantly delayed in group $\mathrm{G}(\mathrm{p}=0.003)$.

Conclusion: We consider that the onset time of lactation is delayed in patients undergoing cesarean section with general anesthesia when compared with patients who undergo cesarean section with spinal and epidural anesthesia and with patients who undergo normal vaginal birth. Because of the delay of awakening and recovery of cognitive functions in general anesthesia, communication between the mother and the newborn is delayed and so is the lactation. (J Turk Ger Gynecol Assoc 2014; 15: 233-8)

Key words: Anesthesia technique, lactation, normal birth

Received: 05 June, 2014

Accepted: 21 October, 2014

\section{Introduction}

Breast milk has many features reinforcing newborn development, and as a nutrient, it is superior to all artificial nutrients (1). Breastfeeding is given great value in the healthy development of a newborn (2). It is important that a mother ideally begins breastfeeding her newborn baby in the first hour after delivery. Cesarean section and primiparity are important risk factors for late onset of breastfeeding (3). Cesarean section is one of the most important operations in obstetrics, and its incidence is approximately $25 \%$ of all deliveries and is progressively increasing. General and regional anesthesia techniques are performed in the anesthetic management of cesarean sections (2).

Consulting the relevant literature, articles about the effects of epidural anesthesia on lactation are common, but there is no article about the effects of general, spinal, and epidural anesthesia techniques and normal vaginal birth on lactation. We designed this study to test our clinical observations about the difference in lactation between patients who underwent cesarean section and vaginal birth.

In our study, we aimed to compare the lactation process by mothers who underwent elective cesarean section under general anesthesia, spinal anesthesia, epidural anesthesia, and normal vaginal birth.

\section{Material and Methods}

The study was approved by the Ethical Committee of Clinical Research from the University of Duzce, Faculty of Medicine (date: 04.08.2011, number: 2011/173). The study was supported by the Committee on Scientific Research Projects from the Düzce University (date: 05.12.2011, number: 2011/350). A total of 84 patients were included into the study, and 6 patients were excluded (Figure 1); 63 of them were underwent elective cesarean section, and 21 patients had a normal vaginal delivery in the Clinic of Gynecology and Obstetrics. All patients were between 18-40 years of age and had a risk of ASA I-II. Before the procedure, informed consent was obtained from all patients (ClinicalTrial.gov ID: NCT02016937).

Exclusion criteria were: non-elective cases, plural pregnancies, preterm pregnancies, fetal anomalies, retardation of fetal development, newborns with birth weight under 2500 grams, infants with a risk of aspiration of meconium or amnions, pathologies affecting acid-base balance, diabetes mellitus, 


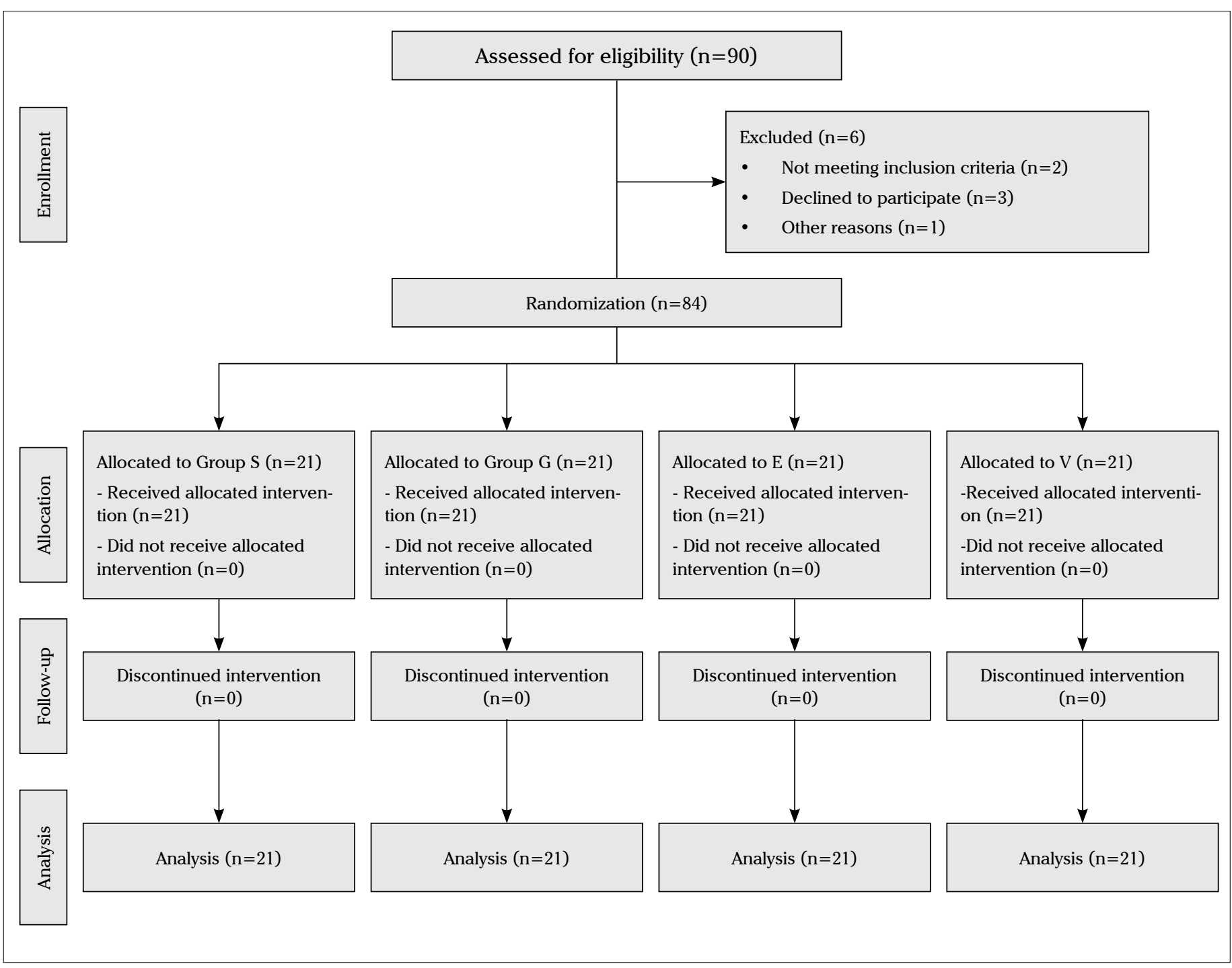

Figure 1. Consolidated Standarts of Reporting Trials (CONSORT) flow diagram of the study

hypertension, antepartum hemorrhage, asthma, COPD (chronic obstructive pulmonary disease), Rhesus incompatibility, obstetric complications (like congenital malformations), history of malignant hyperthermia, morbid obesity, opioid sensitivity, alcohol or drug addiction, diseases of the coronary arteries, congestive heart failure, serious anemia, history of liver or kidney diseases, hypovolemia, hypotension, systemic inflammatory response syndrome, sepsis, history of allergic reactions to drugs used in the study, and history of drugs affecting lactation. Patients were randomly divided into 4 groups: group $\mathrm{G}$ (general anesthesia for cesarean section, $n=21$ ), group $S$ (spinal anesthesia for cesarean section, $n=21$ ), group $E$ (epidural anesthesia for cesarean section, $n=21$ ), and group $V$ (normal vaginal birth without anesthesia, $n=21$ ). A blood sample of 3 milliliters was taken from all patients 2.5 hours before the procedure, and oxytocin and prolactin levels in these blood samples were determined. No patient received premedication. Group G (general anesthesia for cesarean section) received preoxygenation with $100 \%$ oxygen for 3-5 minutes before intubation. In order to expose the fetus minimally to anesthetic agents, induction was performed after the disinfection and covering up of the surgical area. Induction was performed with propofol ( $2 \mathrm{mg} / \mathrm{kg})$ and rocuronium $(0.6 \mathrm{mg} / \mathrm{kg})$. After the onset of neuromuscular block, patients were intubated with compression maneuver of the cricoid cartilage. Controlled ventilation was provided with a Datex Ohmeda $\mathrm{S} / 5$ Avance anesthesia machine with a tidal volume of $8-10 \mathrm{ml} / \mathrm{kg}$ and respiration frequency of $10-12 / \mathrm{min}$. Anesthesia was maintained with oxygen (50\%), air (50\%), and sevoflurane of $1 \mathrm{MAC}$. In the maintenance of anesthesia, rocuronium $0.15 \mathrm{mg} / \mathrm{kg}$ was administered when it was necessary. After delivery of the newborn, fentanyl 1-1.5 mcg/kg was administered. Patients in group $\mathrm{S}$ (spinal anesthesia for cesarean section) received $750-1000 \mathrm{ml}$ of $0.9 \% \mathrm{NaCI}$ solution $(10-15 \mathrm{ml} /$ $\mathrm{kg}$ ) as infusion over 20-30 minutes. Under strict aseptic precautions, a $25 \mathrm{G}$ Quincke spinal needle was introduced into the L3-L4 or L4-L5 intervertebral space in a midline approach in the sitting posture, and after confirmation of free flow of CSF, 10-11 mg of predetermined drug solution (hypertonic bupivacaine 
0.5\%) was injected. We let the operation begin when sensory and motor blockade was verified. Oxygen of 100\% (3 L/min) was administered throughout the operation via nasal cannula. Patients in group E (epidural anesthesia for cesarean section) received $750-1000 \mathrm{ml}$ of $0.9 \% \mathrm{NaCl}$ solution $(10-15 \mathrm{ml} / \mathrm{kg})$ as infusion over 20-30 minutes. We conducted epidural anesthesia with an 18-gauge Tuohy needle at the L3-L4 or L4-L5 epidural space by midline approach in the sitting position. Then, 3-5 minutes after injection of $3 \mathrm{ml}$ lidocaine as a test dose, when the patient had no sign of a subarachnoid injection, like prickling in the lower extremities, or of intravascular injection, like nausea, vomiting, tachycardia, and tinnitus, a 20-gauge epidural catheter was inserted to cephalic. We injected $10 \mathrm{ml}$ of $0.5 \%$ bupivacaine via epidural catheter. The operations began when sensory and motor blockade was verified. Oxygen of 100\% (3 $\mathrm{L} / \mathrm{min}$ ) was administered throughout the operation via nasal cannula.

Patients in all 3 groups were monitored in the operation room with a Datex-Ohmeda monitor, and the electrocardiogram (ECG), systolic blood pressure (SBP), diastolic blood pressure (DBP), mean blood pressure (MBP), heart rate (HR), and peripheral oxygen saturation $\left(\mathrm{SPO}_{2}\right)$ were recorded. Ephedrine 5-10 $\mathrm{mg}$ and/or atropine $0.5 \mathrm{mg}$ was administered when significant hypotension and bradycardia occurred. After delivery of the newborn, $30 \mathrm{IU}$ of oxytocin in a 1000 -cc solution of $0.9 \% \mathrm{NaCI}$ was infused, and if the patient was not hypertensive, methylergonovine $0.2 \mathrm{mg}$ was administered intramuscularly.

Patients in group V (spontaneous vaginal birth without anesthesia) were observed by gynecologists in the Clinic of Gynecology and Obstetrics during the delivery. They received no anesthesia. After delivery of the newborn, 30 IU of oxytocin in a 1000-cc solution of $0.9 \% \mathrm{NaCI}$ was infused, and if the patient was not hypertensive, methylergonovine $0.2 \mathrm{mg}$ was administered intramuscularly.

In all groups, blood samples were taken at the $24^{\text {th }}$ hour after delivery, and oxytocin and prolactin levels were measured. Plasma of blood samples, taken pre- and post-partum, were stored at a temperature of $-80^{\circ} \mathrm{C}$. Prolactin levels were determined with the chemiluminescence immunoassay technique Cobas e 601 kit (Roche ${ }^{\circledR}$ Diagnostics, Mannheim, Germany) using a commercial kit $\left(\right.$ Roche $\left.^{\circledR}\right)$. Oxytocin levels were determined with a commercial ELISA kit (Cusabio Biotech CO. Ltd). All patients' onset times of lactation were recorded. Our primary outcome was lactation onset time. Our secondary outcome was blood prolactin and oxytocin levels.

\section{Statistical analysis}

Data were analyzed using Statistical Packages for the Social Sciences (SPSS, ver. 18.0 for Windows; SPSS Inc. USA). Descriptive statistical methods (average value, standard deviation) were used for evaluation of the data. A power calculation ensured that 21 patients were recruited to provide $80 \%$ power for a $30 \%$ difference in the ratio of patients whose onset of lactation was within 24 hours after delivery either with or without anesthesia techniques at an alpha significance level of 0.05. We used the one-way ANOVA test for quantitative comparison of parameters with normal dispersion, because there were more than two groups, and Tukey test for the determination of the group that was causing the difference. We used independent-samples t-test for comparison of quantitative data and for comparison of parameters with normal dispersion between all groups. Repeated-measures ANOVA was used for comparison of parameters with normal dispersion in the same group, and Bonferroni test was used for determination of the differencecausing quantity. The results were assessed at a confidence interval of $95 \%$. A p value $<0.05$ was considered to be statistically significant, and $\mathrm{p}<0.01$ was highly significant.

\section{Results}

Following approval from the institutional ethical committee, written informed consent from 84 patients was obtained. Mean age was 27.80 years, and mean weight was $75.42 \mathrm{~kg}$, and there were no significant differences in age, weight, and duration of pregnancy between groups (Table 1). In all groups, there was a significant difference between APGAR scores in the 1st and 5th minutes $(\mathrm{p}<0.001)$. The data in Table 1 and the conditions that affected the time of lactation were similar in each group; so, they were not taken into consideration.

There was no significant difference in the prepartum period prolactin levels among all groups $(p=0.350)$. In the postpartum period, prolactin levels were significantly higher in group $\mathrm{G}$ compared to the other groups $(\mathrm{p}=0.011)$. In group, $\mathrm{E}$ they were significantly lower in the postpartum period than in group $\mathrm{V}$ and group $\mathrm{G}(\mathrm{p}=0.026)$. Additionally, in group $\mathrm{S}$, prolactin levels were significantly lower in the postpartum period than in group $\mathrm{V}$ and group $\mathrm{G}(\mathrm{p}=0.015)$ (Table 2$)$. There was no significant difference in the prepartum period oxytocin levels among all groups $(p=0.138)$. In group $\mathrm{V}$, they were significantly higher in the postpartum period than in group $G$ and group $S$ $(p=0.012)$. Eventually, postpartum period oxytocin levels in group $E$ were significantly higher in than in group $S(p=0.039)$ (Table 2). Lactation onset time was compared between groups with and without anesthesia. It was determined that the time of lactation was significantly delayed in the general anesthesia group. Between the other three groups, there was no significant difference in the lactation onset time. In the three groups that received anesthesia, the lactation started after the $24^{\text {th }}$ hour in 13 of 63 patients (20.6\%). In group V, the lactation started after the $24^{\text {th }}$ hour in 2 of 21 patients $(9.5 \%)$. When compared within groups, the lactation onset time was later than the $24^{\text {th }}$ hour in

Table 1. Demographic characteristics

\begin{tabular}{|l|c|c|c|c|c|}
\hline & Group G & Group S & Group E & Group V & p value \\
\hline Age & $28.4 \pm 7.0$ & $28.1 \pm 4.2$ & $26.9 \pm 5.1$ & $27.6 \pm 5.5$ & 0.71 \\
\hline Weight (kg) & $74.1 \pm 11.4$ & $77.7 \pm 14.9$ & $74.5 \pm 6.2$ & $73.1 \pm 9.2$ & 0.56 \\
\hline Gestation (week) & $38.3 \pm 1.2$ & $38.5 \pm 1.3$ & $38.4 \pm 1.1$ & $38.6 \pm 1.1$ & 0.88 \\
\hline APGAR $1^{\text {st }}$ min & $8.2 \pm 0.5$ & $8.1 \pm 0.5$ & $8.1 \pm 0.4$ & $8.1 \pm 0.4$ & 0.83 \\
\hline APGAR 5 ${ }^{\text {th }}$ min & $10 \pm 0.2$ & $10.0 \pm 0.0$ & $10.0 \pm 0.0$ & $10 \pm 0.2$ & 0.83 \\
\hline $\begin{array}{l}\text { Group G: general anesthesia group; Group S: spinal anesthesia group; } \\
\text { GroupE: epidural anesthesia group; Group V: normal vaginal birth group }\end{array}$ \\
\hline
\end{tabular}


8 patients in group $\mathrm{G}(38 \%), 3$ patients in group $\mathrm{S}(14.2 \%)$, and 2 patients in group E (9.5\%) (Table 3$)$.

\section{Discussion}

In our study, it was determined that prolactin levels were highest in group $\mathrm{G}$ and that oxytocin levels were highest in group $\mathrm{V}$ (Table 2). The onset time of lactation was delayed in group G (Table 3).

In cesarean sections, general and regional anesthesia techniques may be performed. Recently, regional anesthesia has been preferred because of its advantages, such as enabling decreased transfer of general anesthetics to the newborn, convenience of controlling early and late onset postoperative pain, avoidance of stress response to surgical trauma, maintenance of consciousness, having no risk of aspiration, avoidance of respiratory depression of the newborn, and avoidance of atonic uterus. Additional advantages of regional anesthesia are that patients can see their baby right after birth and begin breastfeeding in a very short time (4). The most important disadvantages of regional anesthesia are allergic and toxic reactions to local anesthetics, inadequate analgesia, and increased practice time, and it may cause surgical difficulties, headache, lumbar pain, and hypotension. The advantages of general anesthesia are faster induction, less hypotension, less cardiovascular depression, and easier control of airway and respiration (5). In our clinic, we perform general, spinal, or epidural anesthesia according to the patient's demand, the presence of additional diseases, and urgency of the case.

There is no doubt that breastfeeding has a vital necessity for a newborn's health. Breastfeeding is affected by many factors, like the mother's feeding demands, regional traditions, the relationship between the mother and the newborn, education, social factors, type of delivery, and duration of delivery. Because of these factors, the type of anesthesia can play a role on onset time of breast feeding. In the literature, there is no study about the effects of different anesthesia techniques on the beginning time of lactation. So, we think that our study could enlighten future studies. The effects of different types of analgesics and the differentiation of systemic and neuroaxial anesthesia could not be determined. In a few studies, the side effects of epidural anesthesia on breastfeeding were compared between patients undergoing epidural anesthesia and patients without anesthesia, and no negative effect of epidural anesthesia was observed (6).

In one study from the literature, bupivacaine and fentanyl were used for epidural anesthesia, and another group received no anesthesia (7). The beginning time of lactation was determined. Similarly, Chang and Heaman (8) established two groups with and without epidural anesthesia, and no significant difference between the groups was determined in either study. In another study that was found, a significant difference in the lactation onset time was determined between cesarean section patients undergoing regional and general anesthesia, but there was no difference between the epidural anesthesia and no-anesthesia groups (9). In our results, no significant difference in the lactation onset time was determined between the epidural and spinal anesthesia and vaginal birth groups. However, in the general anesthesia group, lactation onset time was significantly delayed compared to other groups $(\mathrm{p}=0.003)$. The most significant difference was between the groups of general and spinal anesthesia $(p=0.006)$. In the same study, onset of lactation was significantly later in the general anesthesia group (9). In all three studies, the groups of epidural anesthesia and no anesthesia were compared, and it was determined that the onset time of lactation was the latest in the epidural group (10-12). Especially, the breastfeeding rate in the first 4 hours was determined to be lower in mothers who delivered with epidural anesthesia (13). These newborns needed synthetic nutrients, and in addition, a lower

Table 2. Comparison of hormone levels pre- and post-partum between groups. Oxytocin levels were defined as pg/mL, and prolactin levels were defined as $\mathrm{ng} / \mathrm{mL}$

\begin{tabular}{|l|c|c|c|c|c|}
\hline & Group G & Group S & Group E & Group V & p value \\
\hline Prolactin $_{\text {pre }}$ & $244.6 \pm 105.7$ & $198.2 \pm 57.4$ & $222.3 \pm 81.9$ & $237.1 \pm 103.5$ & 0.350 \\
\hline Prolactin $_{\text {post }}$ & $363.7 \pm 120.9$ & $270.4 \pm 100.4^{\mathrm{a}}$ & $264.0 \pm 108.2^{\mathrm{b}}$ & $300.8 \pm 87.5$ & 0.015 \\
\hline Oxytocin $_{\text {pre }}$ & $2.3 \pm 0.2$ & $2.2 \pm 0.4$ & $2.4 \pm 0.5$ & $2.4 \pm 0.3$ & 0.138 \\
\hline Oxytocin $_{\text {post }}$ & $2.3 \pm 0.3$ & $2.2 \pm 0.5$ & $2.6 \pm 0.7^{\mathrm{d}}$ & $2.8 \pm 0.4^{\mathrm{c}}$ & 0.012 \\
\hline
\end{tabular}

Group G: general anesthesia group; Group S: spinal anesthesia group; Group E: epidural anesthesia group; Group V: normal vaginal birth group; Pre: preoperative; post: postoperative

agroup S significantly lower than group V and group G, bgroup E significantly lower than group V and group G, cgroup V significantly higher than group $\mathrm{G}$ and group $\mathrm{S}$, 'group $\mathrm{E}$ significantly higher than group $\mathrm{S}$

Table 3. Comparison of lactation onset time among groups

\begin{tabular}{|l|c|c|c|c|c|}
\hline & Group G & Group S & Group E & Group V & p value \\
\hline Lactation onset time (hour) & $25.0 \pm 22.9^{\mathrm{a}}$ & $10.8 \pm 10.2$ & $11.8 \pm 8.8$ & $10.9 \pm 9.7$ & 0.003 \\
\hline agroup G significantly higher than the other groups \\
Group G: general anesthesia group; Group S: spinal anesthesia group; Group E: epidural anesthesia group; Group V: normal vaginal birth group \\
\hline
\end{tabular}


proportion of them was breastfeeding only when discharged. In the literature, it was reported that epidural analgesia enhances postpartum analgesia and mental condition and that it has positive effects on breastfeeding (14). In our study, no significant difference was determined between the epidural anesthesia and vaginal birth groups in lactation onset time.

In one study, prolactin, estrogen, and ACTH levels before and after cesarean section were determined in 17 patients. Three different anesthetics were used before general and epidural anesthesia; 9 women could breastfeed, and when compared with the other patients, their prolactin levels were increased, and their ACTH and estrogen levels were decreased (15). There was no significant difference in hormone levels and lactation between anesthetic techniques. In our study, there was no significant difference in prepartum hormone levels, but we determined that postpartum prolactin levels were significantly higher in group $\mathrm{G}$ than in the other groups. So, the results of this study were not consistent with ours.

Prolactin levels and lactation onset times were compared between women undergoing epidural analgesia and women who had a vaginal birth without anesthesia (16). Prolactin levels in the epidural group were significantly lower, but there was no significant difference in lactation-relevant parameters. In addition, it was determined in this study that prolactin arrived at the peak level in the second hour and persisted at that peak level for 24 hours (16). In our study, prolactin levels were lower in the epidural anesthesia group than in the vaginal birth group but not statistically significantly (Table 2). We thought that the cause of the higher prolactin levels in the general anesthesia group than in the spinal anesthesia group could be the difference between the effects of the anesthetic agents used in both anesthesia techniques. As is known, many drugs can affect prolactin levels. These effects occur more often with systemic usage of drugs, and it could increase prolactin levels. Increasing oxytocin, prolactin, and betaendorphin levels can affect the emotional state of the mother, the relationship between the mother and the newborn, and lactation (17). Oxytocin and prolactin increase the production and secretion of breast milk all throughout the breastfeeding period. Oxytocin plays an important role in the contraction of the uterus by vaginal birth. It was determined that oxytocin and prolactin levels increase significantly during lactation. Basic levels of hormones were determined to be especially high between the 4th day and 3rd or 4th month (18). In studies that included a limited number of patients and in which only oxytocin levels in normal delivering women were measured, it was determined that there was no relationship between oxytocin levels and lactation but that only multiparity had a positive correlation with oxytocin. In these two studies, no different anesthesia techniques were used, and only oxytocin levels were evaluated in normal delivering women (19-20). One of them evaluated oxytocin levels in patients undergoing cesarean section with spinal anesthesia and made a comparison between patients that had contractions and the ones who had no contractions (20). They determined no significant difference in oxytocin levels in blood serum, cerebrospinal fluid serum, and umbilical cord serum between both groups. In our study, no significant difference between prepartum oxytocin levels was determined, but there were differences in postpartum oxytocin levels among the four groups. Oxytocin levels were especially lower in the groups undergoing general and spinal anesthesia. In oxytocin levels compared between the epidural and normal birth groups, there was no significant difference, and the levels were significantly increased compared with the other two groups. It has to be taken into consideration that oxytocin is playing an important role in lactation and uterine contractions. Because of the similar effects of epidural anesthesia on hormone levels during normal birth, it is more rational to prefer epidural anesthesia (20). We determined that the most significant difference in prolactin levels was between groups $\mathrm{G}$ and $\mathrm{S}$, and eventually, the most significant difference in lactation onset time was among groups $\mathrm{G}, \mathrm{S}$, and $\mathrm{E}$. As a result of these considerations, spinal and epidural anesthesia may have advantages over general anesthesia.

We observed that lactation onset time is delayed in patients undergoing cesarean section with general anesthesia when compared with patients undergoing cesarean section with spinal and epidural anesthesia and normal birth. Because of the delay in awakening and in the recovery of cognitive functions in general anesthesia, communication between the mother and the newborn is delayed and so, too, is lactation. General anesthetic drugs may have an effect on brain stimulation of the breast milk secretion process and the onset of lactation.

Ethics Committee Approval: Ethics committee approval was received for this study from the ethics committee of Düzce University Faculty of Medicine.

Informed Consent: Written informed consent was obtained from patients who participated in this study.

Peer-review: Externally peer-reviewed.

Author contributions: Concept - L.K., I.S.S., Y.D.; Design - L.K., Y.D.; Supervision - G.S., S.A.K.; Resource - Y.D.; Materials - L.K., I.K.; Data Collection\&/or Processing - L.K., O.E.; Analysis\&/or Interpretation - L.K., Y.D.; Literature Search - L.K., I.S.S.; Writing - I.S.S., Y.D.; Critical Reviews - I.S.S., Y.D., O.E.

Conflict of Interest: No conflict of interest was declared by the authors.

Financial Disclosure: The study was supported by the Committee on Scientific Research Projects from the Düzce University.

\section{References}

1. Work Group on Breastfeeding. American Academy of Pediatrics. Breastfeeding and the use of human milk. Pediatrics 1997; 100: 1035-92. [CrossRef]

2. Arlotti JP, Cottrell BH, Lee SH, Curtin JJ. Breast feeding among low-income women with and without peer support. J Community Health Nurs 1998; 15: 163-78. [CrossRef]

3. Jones E, Spencer SA. The physioloji of lactation. Paediatr Child Health 2007: 17: 244-8. [CrossRef]

4. Volmanen P, Valanne J, Alahuhta S. Breast-feeding problems after epidural analgesia for labour: a retrospective cohort study of pain, obstetrical procedures and breast-feeding practices. Int $\mathrm{J}$ Obstet Anesth 2004; 13: 25-9. [CrossRef]

5. Morgan GE, Maged MS, Michael MJ. Clinical anesthesiology. 2006. p.890-907. 
6. Baumgarder DJ, Muehl P, Fischer M,Pribbenow B. Effect of labor epidural anesthesia on breast-feeding of healthy full-term newborns delivered vaginally. J Am Board Fam Pract 2003; 16: 7-13. [CrossRef]

7. Radzyminski S. The effect of ultra low dose epidural analgesia on newborn breastfeeding behaviors. J Obstet Gynecol Neonatal Nurs 2003; 32: 322-31. [CrossRef]

8. Chang ZM, Heaman MI. Epidural analgesia during labor and delivery: effects on the initiation and continuation of effective breastfeeding. J Hum Lact 2005; 21: 305-14. [CrossRef]

9. Albani A, Addamo P, Renghi A, Voltolin G, Peano L, Ivani G. The effect on breastfeeding rate of regional anesthesia technique for cesarean and vaginal childbirth. Minerva Anestesiol 1999; 65: 625-30.

10. Henderson JJ, Dickinson JE, Evans SF, McDonald SJ, Paech MJ. Impact of intrapartum epidural analgesia on breast-feeding duration. Aust N Z J Obstet Gynaecol 2003; 43: 372-7. [CrossRef]

11. Volmanen P, Valanne J, Alahuhta S. Breast-feeding problems after epidural analgesia for labour: a retrospective cohort study of pain, obstetrical procedures and breast-feeding practices. Int $\mathrm{J}$ Obstet Anesth 2004; 13: 25-9. [CrossRef]

12. Wilson MJA, MacArthur C, Shennan A. The effect of epidural analgesia on breast feeding: Analysis of a randomized controlled trial. Anaesthesia 2010; 65: 145-53. [CrossRef]
13. Wiklund I, Norman M, Uvnäs-Moberg $\mathrm{K}$, Ransjö-Arvidson $\mathrm{AB}$, Andolf E. Epidural analgesia: Breast-feeding success and related factors. Midwifery 2009; 25: 31-8. [CrossRef]

14. Wang BP, Li QL, Hu YF. Impact of epidural anesthesia during delivery on breast feeding. Di Yi Jun Yi Da Xue Xue Bao 2005; 25: 114-5.

15. Siegismund K, Justus B, Schollberg K. Behavior of prolactin, estradiol and ACTH in maternal blood in delivery by cesarean section. Zentralbl Gynakol 1986; 108: 212-9.

16. Chen YM, Li Z, Wang AJ, Wang JM. Effect of labor analgesia with ropivacaine on the lactation of paturients. Zhonghua Fu Chan Ke Za Zhi 2008; 43:502-5.

17. Lennart Righard. Making childbirth a normal process. Birth 2001; 28: 1-4. [CrossRef]

18. Uvnäs-Moberg $\mathrm{K}$, Widström $\mathrm{AM}$, Werner $\mathrm{S}$, Matthiesen $\mathrm{AS}$, Winberg J. Oxytocin and prolactin levels in breast-feeding women. Correlation with milk yield and duration of breast-feeding. Acta Obstet Gynecol Scand 1990; 69: 301-6. [CrossRef]

19. Lucas A, Drewett RB, Mitchell MD. Mitchell Breast-feeding and plasma oxytocin concentrations. Br Med J 1980; 281: 834-5. [CrossRef]

20. Sayyah Melli M, Alizadeh M, Rahbani-noubar M. Maternal and neonatal oxytocin levels during cesarean section with or without labor pain and puerperal complications. Int J Endocrinol Metab 2006; 4: 188-94. 